\title{
IDENTIFICACIÓN DE CONSTRUCTOS \\ COMPETITIVOS Y ESTRATÉGICOS DE LAS \\ EXPORTACIONES DE MANZANAS CHILENAS \\ A LOS MERCADOS ASIÁTICOS ${ }^{1}$
}

\author{
CRISTIAN ADASME-BERRIOS², RUBÉN FRANCISCO ORTEGA-ROJAS², \\ MARCELO RODRÍGUEZ-GUTIÉRREZ², GERMÁN LOBOS-ANDRADE
}

RESUMEN - En esta investigación se analiza la competitividad de las exportaciones de manzanas de la región del Maule, Chile, en los mercados asiáticos de China, Hong Kong, Taiwán e India. El objetivo fue identificar estrategias competitivas y determinar las variables de ventaja competitiva de mayor relevancia. Para determinar los constructos estratégicos se realizó un bootstrap junto con un análisis factorial de componentes principales (PCA). Los constructos encontrados indican que las estrategias competitivas del sector exportador está orientado en lograr economías de escalas y optimizar procesos productivos y comerciales, para lograr menores costos y competir eficientemente en los mercados asiáticos.

Términos de Indexación: competitividad, estrategia competitiva, análisis factorial.

\section{IDENTIFICATION OF COMPETITIVE AND STRATEGIC CONSTRUCTS TO THE CHILEAN APPLES IN ASIATIC MARKETS}

\begin{abstract}
The research analyzes the competitiveness of apples exportations from Maule's Region, Chile, toward Asian markets of China, Hong Kong, Taiwan and India. The objective was to identify competitive strategies and determine the most important competitive advantage variables. A bootstrap and a factor analysis of principal component analysis (PCA) were carried out determining the strategic constructs. The results found indicate that the competitive strategies of the export sector is focused on achieving economies of scale and optimize production and trade, obtaining lower costs and effective competition in Asian markets.
\end{abstract}

Index terms: Competitiveness, competitive strategy, factorial analysis.

\section{INTRODUCCIÓN}

Según Schwab (2010) la competitividad es el conjunto de las instituciones, políticas y factores que determinan el nivel de productividad de un país, el que a su vez establece el nivel sostenible de prosperidad que puede ser percibido por una economía. Para lograr dicha competitividad se debe generar una ventaja competitiva, que es una función que puede brindar el mismo valor de forma más eficiente que los competidores, o realizar actividades que agreguen un valor único que los clientes prefieran ante la competencia (GARCÍA; SERRANO, 2003; PORTER, 2006). Lo anterior permite desglosar la estrategia genérica que puede desarrollar una empresa en un mercado, la cual puede ser de "liderazgo en costos", "diferenciación" o la derivación de ambas en un "enfoque" (PORTER,
1982; 2006; THOMPSON; STRICKLAND, 1994). Cuando una empresa intenta alcanzar todas las estrategias genéricas al mismo tiempo, corre el riesgo de quedar "pegado en el medio", posición donde pierde competitividad (PORTER, 1982). Por otra parte, las empresas también pueden alcanzar el éxito generando bajo costo y diferenciación, gracias a las tecnologías de gestión de la calidad y flexibilidad productiva (FJELDSTAD; HAANAES, 2001; LEITNER; GÜLDENBERG, 2010). En el ámbito del comercio internacional, estos fundamentos son esenciales para ser competitivos y en el caso de Chile, está posicionado como uno de los principales exportadores de fruta fresca y específicamente en manzanas. Steward (2008) plantea que Chile es de los más eficientes productores del mundo y actualmente es líder mundial en competitividad en la industria de manzanas (BELROSE, 2010). En cuanto a mercados mundiales, el asiático es el que

\footnotetext{
'(Trabalho 076-11). Recebido em: 08-02-2011. Aceito para publicação em: 29-03-2012.

${ }^{2}$ Universidad Católica del Maule, Facultad de Ciencias Agrarias y Forestales, Casilla 7D, Curicó, Chile. E-mails: cadasme@ucm.cl, ruben.ortega.1125@alu.ucm.cl; mrodriguez@ucm.cl

${ }^{3}$ Universidad de Talca, Facultad de Ciencias Empresariales, Casilla 721, Talca, Chile. E-mail: globos@utalca.cl
} 
ha crecido mayormente en los últimos años, debido al incremento del producto interno bruto y de sus tasas de crecimiento, lo que implicó un aumento en la demanda por productos como alimentos básicos para estas economías. Se estima que un $25 \%$ de las importaciones de frutas y verduras del mundo provienen de mercados emergentes como China e India, con cifras que siguen en aumento, con un crecimiento de 5 a 10 veces mayor que el mercado europeo (REARDON, 2007). La existencia de diferentes competidores de Chile en el mercado de la manzana, los cuales cuentan con estrategias de diferenciación por calidad y homogeneidad de la fruta que exportan, hace necesario definir las estrategias competitivas de Chile hacia los mercados emergentes de Asia. Por tal razón, los objetivos de la investigación son identificar las estrategias que presenta el sector exportador de manzanas de la Región del Maule y determinar su relación con las estrategias genéricas y cuáles son las variables competitivas de mayor relevancia para así enfrentar correctamente el desafío del mercado asiático.

\section{MATERIALES Y MÉTODOS}

El estudio contempla un diseño de investigación de tipo exploratorio-descriptivo y se realizó con empresas de la Región del Maule que son exportadoras de manzanas al mercado asiático. Se aplicó un cuestionario estructurado y específico para la investigación a los gerentes comerciales de $14 \mathrm{em}$ presas durante la temporada agrícola 2009/2010. La determinación de la muestra consistió en identificar las empresas que exportan manzanas a China, Hong Kong, Taiwán e India, por medio de la consulta a la base de datos de Decofrut con fecha 14 mayo del 2010, de las cuales 35 exportadoras pertenecen a la Región del Maule, resultando un tamaño muestral $\mathrm{n}=14$ con un nivel de confianza del $95 \%$ y un 5\% de margen de error (HERNÁNDEZ et al. 2006). La encuesta se dividió en cuatro secciones para un total de 119 variables en escala Likert de cinco niveles (KINNEAR; TAYLOR, 2009). La definición de las secciones y las variables, se basan en los estudios de Porter (1982); Chacko et al. (1997) y Villacura (2001). Frente a la problemática de tener un número bajo de la muestra $(\mathrm{n}=14)$ para realizar un análisis factorial de componentes principales (PCA), se utilizó el método bootstrap (EFRON, 1979) para realizar un remuestreo de las bases de datos originales. Esta técnica de simulación utiliza una y otra vez las observaciones originales para constituir un universo del cual extraer repetidas muestras. A partir de una población $\mathrm{P}=\left\{\mathrm{X}_{1}, \mathrm{X}_{2}, \ldots . \mathrm{X}_{\mathrm{n}}\right\}$ donde la po- blación corresponde a 35 exportadoras, es extraída una muestra $S_{1}^{*}=\left\{X_{1}, X_{2}, \ldots . X_{n}\right\}$ de tamaño $S=14$, determinado estadísticamente, de la cual es extraída una nueva muestra bootstrap $\mathrm{S}_{\mathrm{b}}{ }^{*}=\left\{\mathrm{X}_{1}{ }^{*}, \mathrm{X}_{2}{ }^{*}, \ldots\right.$. $\left.\mathrm{X}_{\mathrm{n}}^{*}\right\}$, por medio de un muestreo con reposición (KARANTININIS et al., 2010). La simulación puede ser a partir de muestras de 10 a 20, obteniendo buenos resultados y se considera inadecuada según Chernick (1999), para muestras inferiores a 5. Los datos originales eran $\mathrm{S}=14$ y las muestras regeneradas con el programa XLSTAT 2010 fueron $\mathrm{S}_{\mathrm{b}}=300$, que representa el tamaño óptimo del índice KaiserMeyer-Olkin (KMO), al menos de 0,5 para cumplir con los principios de interpretabilidad y parsimonia de los factores (BISQUERRA, 1989; LUQUE, 2000). Además, muestras bootstrap de $\mathrm{S}_{\mathrm{b}}=100$ a 500 son satisfactorias para los análisis de datos (WEHRENS et al., 2000). Luego fue realizado un análisis de fiabilidad reduciendo las variables a 57, a partir de 119 originales. Posteriormente se realizó un PCA que permitió transformar un conjunto de variables intercorrelacionadas, en otros no correlacionados denominados factores [1] (LUQUE, 2000). El primer factor es la combinación que explica la mayor parte de la varianza y el segundo factor es aquel que de forma ortogonal al primero explica la segunda mayor variabilidad. Posteriormente, se aplicó una rotación ortogonal varimax con Kaiser (1958) para extraer los componentes subyacentes en las variables de estudio. Para realizar este análisis se utilizó el programa SPSS versión 18.0 (SPSS INC., CHICAGO IL. USA).

$$
X_{i j}=a_{1 j} F_{i 1}+a_{2 j} F_{i 2}+\ldots . .+a_{k j} F_{i j}
$$

Donde $\mathrm{X}_{\mathrm{ij}}$ representa al valor de la $\mathrm{j}$ ésima variable correspondiente al i ésimo caso, $\mathrm{a}_{\mathrm{ij}}$ corresponde a las puntuaciones factoriales y $\mathrm{F}_{\mathrm{ij}}$ representa los coeficientes factoriales correspondientes al i ésimo caso.

\section{RESULTADOS Y DISCUSIONES}

El $57,1 \%$ de las exportadoras pertenecen al segmento de las grandes empresas, ya que tienen ventas superiores a US $\$ 4.500 .000$ anuales, según la clasificación chilena. En cuanto al volumen de exportación, el $64,3 \%$ de las grandes empresas exporta al mundo menos de 1.000 .000 de cajas anuales y el $35,7 \%$ sobre esta cifra. Con destino a los mercados asiáticos el 71,4\% exporta menos de 300.000 cajas y el $28,6 \%$ sobre esta cifra. El 57,2\% de las empresas tiene presencia exportadora en tres y/o cuatro de los mercados estudiados y el $42,8 \%$ 
en uno y/o dos de estos. Además, el 78,6\% de las exportadoras tiene menos de 10 años de experiencia exportando hacia estos mercados y el $21,4 \%$ restante más de 10 años.

\section{ternas locales}

Análisis de las variables competitivas ex-

El PCA agrupó 10 variables en dos componentes, con un porcentaje acumulado de la varianza explicada del $61,45 \%$ con rotación varimax de tres iteraciones. Un índice KMO de 0,607; un Alfa de Cronbach de 0,759; junto con una Prueba de esfericidad de Bartlett con una significancia $<0,001$; datos que indican la factibilidad del PCA (Tabla 1).

Factor 1. Este factor explica el 39,93\% de la varianza y está correlacionado con las variables "disponibilidad tecnológica", "políticas internacionales", "disponibilidad de suministros y servicios", "estabilidad económica del país", "sistemas de información tecnológica y comercial", "infraestructura portuaria nacional" y las "instituciones gubernamentales". Este factor fue denominado "Plataforma competitiva" debido a que los resultados concuerdan en parte con la clasificación realizada por Schwab (2010). Dado que este factor incluye los tres primeros pilares de la competitividad, "Instituciones", "Infraestructuras", y "Estabilidad macroeconómica", solo faltando el cuarto pilar (Salud y Educación) para formar los requerimientos básicos de la competitividad. Además, contiene el noveno pilar "Disponibilidad tecnológica" que junto con los anteriores impulsan la economía de un país (SCHWAB; PORTER, 2008), resultados que concuerdan con Santana (2004) en cuanto a variables competitivas sistémicas. También hay que señalar que en las políticas comerciales existe una visión positiva, lo que concuerda con el actual posicionamiento del país en el mercado mundial, reflejado en la incorporación de Chile a la OCDE (Organización para la Cooperación y Desarrollo Económico) el año 2010.

Factor 2. Este factor explica el $21,51 \%$ de la varianza y agrupa las variables "sofisticación de las redes de negocios", "costos de las materias primas, suministros y servicios en el país", junto con la "eficiencia y aptitud productiva del sector exportador de manzanas". Este factor fue denominado "Externalidad productiva" debido a que en el análisis del constructo se aprecia una visión positiva de las redes de negocios del país y de la aptitud productiva, que denotan una economía con una alta sofisticación acorde a las exigencias de los mercados mundiales y que permiten fomentar la eficiencia dentro de la cadena comercial; a través de la reducción de costos. Pero existe una correlación negativa de la variable que contiene los costos de insumos y de servicios dentro del país, por la dificultad de contar con servicios de bajo costo, producto del aumento de precio cada año. Resultados que concuerdan en parte con el estudio de Chacko et al. (1997) y difieren de los resultados obtenidos por Mamaqui et al. (2002) quienes señalan que los factores competitivos de entorno más relevantes, corresponden a la competencia del mercado, competencia empresarial y retos de los productos.

\section{Análisis de las variables competitivas ex- ternas globales}

El PCA agrupó 10 variables y arrojó dos componentes con un porcentaje acumulado de la varianza explicada del $59,21 \%$ y con rotación varimax de seis iteraciones. Un índice KMO de 0,561 ; un Alfa de Cronbach de 0,648; junto con una Prueba de esfericidad de Bartlett con una significancia $<0,001$; datos que indican la factibilidad del PCA (Tabla 2).

Factor 1. Este factor explica el $32,33 \%$ de la varianza y se correlaciona con las variables "exigencias de normativas de producción internacionales", "exigencias fitosanitarias de los mercados de destino", "tasas arancelarias y barreras para arancelarias", "políticas de los tratados internacionales", "efectos de los tratados de libre comercio" y las "carteras de clientes". Este factor fue denominado "Comercio exterior" ya que está constituido por las barreras que tiene el sector exportador para ingresar a los mercados asiáticos. Pero, estas barreras no son limitantes para el sector exportador chileno, producto de los Tratados de Libre Comercio (TLC) con China, Corea del Sur y Japón, además del acuerdo parcial con India que significa que Chile tiene beneficios y ventajas para entrar a estos mercados, por la reducción progresiva de aranceles aduaneros y de las barreras para arancelarias. Sin embargo estos resultados difieren de los hallazgos encontrados por Lobos (2007) y Lobos \& Petri (2008), quienes señalan que existen otros factores que influyen en el comercio exterior, como son el efecto del tipo de cambio y la volatilidad de los precios FOB de las frutas en los mercados internacionales.

Factor 2. Este factor explica el $26,87 \%$ de la varianza y está correlacionado con las variables "estabilidad económica del mercado asiático", "oferta de competidores americanos (EE.UU., Brasil y Argentina)", "demanda internacional (asiática)" y la "oferta de competidores asiáticos (China, India, Japón)". Este factor fue denominado "Mercado asiático" porque presenta una correlación positiva en las variables de estabilidad económica del mercado asiático y demanda asiática, debido a sus 
altas tasas de crecimiento económico y de su población, junto con la demanda creciente por frutas. No obstante, existe una correlación negativa en la variable de oferta de los competidores americanos, específicamente de EE.UU., ya que es el principal competidor de Chile en los mercados asiáticos, por volúmenes, calidad y participación. También existe correlación negativa por la variable oferta asiática, producto de un incremento en la oferta de manzanas proveniente de los principales productores de Asia, lo que podría disminuir la demanda del producto. Estos resultados son consistentes con los hallazgos obtenidos por Belrose (2010).

\section{Análisis de las variables competitivas in- ternas}

El PCA agrupó 13 variables en dos componentes, con un porcentaje acumulado de la varianza explicada del $59,64 \%$ y con rotación varimax de tres iteraciones. Un índice KMO de 0,589; un Alfa de Cronbach de 0,839; junto con una Prueba de esfericidad de Bartlett con una significancia $<0,001$; datos que indican la factibilidad del PCA (Tabla 3).

Factor 1. Este factor contiene el $32,51 \%$ de la varianza explicada y agrupó las variables "sistemas de conducción y poda" "políticas internas de producción limpia", "sistemas de control de calidad", "sistemas de optimización de servicios y suministros", "sistemas de control de inocuidad alimentaria", "integración vertical hacia atrás" y "sistemas de mejoramiento de la productividad". Este factor fue denominado "Optimización productiva" debido a que existe una visión positiva frente a las acciones llevadas a cabo por el sector exportador para mejorar la producción de manzanas en Chile, con manejos técnicos de la conducción y poda que logra una mayor eficiencia en la utilización de zonas productivas del árbol frutal. Pero no sólo es una búsqueda de más productividad, también existe preocupación de integrar la calidad alimentaria en el proceso productivo, incorporando aspectos de inocuidad alimentaria por medio de normativas internacionales y estándares propios del sector. Resultados que concuerdan en parte con los hallazgos encontrados por Tapia \& Cooper (2006) y Contreras (2001). Mientras que Orellana (2009) difiere de los resultados, dado que señala que los factores productivos tienen menos importancia en comparación a las condiciones climáticas y geográficas que tiene Chile (Ventajas Comparativas).

Factor 2. Este factor explica el 27,12\% de la varianza y está constituido por las variables "almacenaje", "tamaño e infraestructuras de la empresa", "líneas de embalaje", “capacitación laboral", "automatización de los procesos producti- vos y comerciales" y "sofisticación de las redes de negocios". Este factor fue denominado "Capacidad industrial" ya que en el análisis de este constructo existe correlación positiva frente al desarrollo de activos fijos, como infraestructura, líneas de procesos y bodegaje del sector exportador en la Región del Maule. Este es un aspecto indispensable de una industrialización moderna, con capacidad de procesamiento y de almacenaje adecuado, para satisfacer los requerimientos de demanda de un producto. Además, otro aspecto importante son los recursos humanos, reflejados en la capacitación laboral, con actualización y perfeccionamiento del personal, que permiten una modernización a la par con la infraestructura y tecnologías de procesamiento y almacenaje. Estos resultados concuerdan con los estudios preliminares de Durán \& Silva (1996), Villacura (2001), Rojas (2007), pero no necesariamente con los resultados encontrados por Tapia \& Cooper (2006), quienes señalan que los aspectos competitivos relevantes son la mantención y mejoramiento de la calidad del producto, la investigación genética y varietal en manzanas y la protección de los derechos de propiedad intelectual de las innovaciones.

\section{Análisis de las estrategias competitivas}

El PCA agrupó las 12 variables en dos componentes, con un porcentaje acumulado de la varianza explicada del $62,42 \%$ y con rotación varimax de tres iteraciones. Un índice KMO de 0,562; un Alfa de Cronbach de 0,879; junto con una Prueba de esfericidad de Bartlett con una significancia $<0,001$; datos que indican la factibilidad del PCA (Tabla 4).

Factor 1. Este constructo contiene el 31,26\% de la varianza explicada, constituido por las variables "mano de obra", "especialización", "control de finanzas", "reducción de costos", "integración vertical" y "control del flujo de productos". Este factor fue denominado "Optimización de costos" porque agrupa aquellas variables estratégicas que impactan en la reducción de costos dentro de una industria, donde la más relevante es la mano de obra, ya que los trabajadores son el principal costo para la industria frutícola y es primordial su optimización para ser más competitivos. Resultado que concuerda con la investigación de Mamaqui et al. (2002) quienes plantean que las estrategias del sector agroindustrial en España son los recursos humanos, eficiencia de la producción, conocimiento y gestión. A diferencia de Villacura (2001), que indica que el sector exportador de manzanas de Chile tiende a proponer una estrategia competitiva basada en la obtención de menores costos de producción mediante economías de escala.

Factor 2. Contiene el $31,16 \%$ de la varianza explicada y está compuesto por las variables "ca- 
nales de distribución", "servicios", "estrategias de costos", "estrategias de compras", "mercados objetivos", "relaciones comerciales con empresas matrices" y "estrategias de ventas". Este factor fue denominado "Gestión comercial" debido a que agrupa variables de administración y comercio del sector exportador, destacando la variable canales de distribución del producto, punto crítico en la exportación de manzanas a los mercados asiáticos, ya que dentro de la logística es necesario tener en cuenta la distancia y tiempo de llegada en las fechas exactas a los mercados. Por ende, la formulación de estrategias y elección de los canales de comercialización se debe realizar pensando en buscar los canales más expeditos. Resultados que concuerdan con Mamaqui et al. (2002) en cuanto a la estrategia de gestión. Pero Chacko et al. (1997) plantean que la agroindustria de EE.UU. considera que los factores competitivos relevantes son la "calidad", "velocidad de entrega", "flexibilidad" y "costos". En cambio Tapia (2006), difiere de nuestros resultados proponiendo "posicionamiento mundial" y "participación en los mercados" como los más importantes.

TABLA 1- Análisis de componentes principales de las variables competitivas externas a nivel local

\begin{tabular}{|c|c|c|}
\hline \multirow[b]{2}{*}{ Variables } & \multicolumn{2}{|c|}{ Componente } \\
\hline & $\begin{array}{l}\text { Plataforma } \\
\text { competitiva }\end{array}$ & $\begin{array}{c}\text { Externalidad } \\
\text { productiva }\end{array}$ \\
\hline 1. Disponibilidad tecnológica & $\mathbf{0 , 8 5 7}$ & $\mathbf{0 , 2 3 8}$ \\
\hline 2. Políticas internacionales & $\mathbf{0 , 8 3 3}$ & $-0,143$ \\
\hline 3. Disponibilidad de suministros y servicios & 0,824 & $-0,093$ \\
\hline 4. Estabilidad económica del país & 0,744 & 0,124 \\
\hline 5. Sistemas de información tecnológica y comercial & 0,709 & 0,114 \\
\hline 6. Infraestructura portuaria nacional & 0,580 & 0,253 \\
\hline 7. Instituciones gubernamentales & $\mathbf{0 , 5 7 1}$ & $\mathbf{0 , 3 3 0}$ \\
\hline 8. Sofisticación de las redes de negocios & 0,067 & 0,935 \\
\hline 9. Costos de materias primas, suministros y servicios en el país & 0,056 & $-0,802$ \\
\hline 10. Eficiencia y aptitud productiva del sector exportador de manzanas & 0,398 & $\mathbf{0 , 5 8 8}$ \\
\hline Porcentaje de la varianza explicada por el factor & $39,93 \%$ & $21,51 \%$ \\
\hline Medida de adecuación muestral de Kaiser-Meyer-Olkin. & \multicolumn{2}{|c|}{$\mathbf{0 , 6 0 7}$} \\
\hline Alfa de Cronbach & \multicolumn{2}{|c|}{0,759} \\
\hline Prueba de esfericidad de Bartlett. & $\frac{X^{2}}{2177,145}$ & $\begin{array}{l}\text { Sig. } \\
\mathbf{0 , 0 0 0}\end{array}$ \\
\hline
\end{tabular}
ha convergido en 3 iteraciones.

TABLA 2-Análisis de componentes principales de las variables competitivas externas nivel global.

\section{Variables}

1. Exigencias de normativas de producción internacionales

2. Exigencias fitosanitarias de los mercados de destino

3 Tasas arancelarias y barreras para arancelarias

4. Efectos de los tratados de libre comercio

5. Políticas de los tratados internacionales

6. Carteras de clientes

7. Estabilidad económica del mercado asiático

8. Oferta de competidores americanos(USA, Brasil, Argentina)

9. Demanda internacional ( asiática )

10. Oferta de competidores asiáticos(China, Japón, India)

Porcentaje de la varianza explicada por el factor

Medida de adecuación muestral de Kaiser-Meyer-Olkin.

\section{Componente}

Comercio exterior Mercado asiático

$0,876 \quad-0,270$

$0,743-0,255$

$\mathbf{0 , 7 3 9}-\mathbf{0 , 1 4 0}$

$0,700 \quad 0,461$

$\mathbf{0 , 6 4 7} \quad \mathbf{0 , 5 7 7}$

$0,605 \quad 0,294$

$-0,239 \quad 0,846$

$0,149-0,706$

$0,046 \quad 0,630$

$-0,104 \quad-0,537$

$32,33 \% \quad 26,87 \%$

\begin{tabular}{cccc}
\hline Medida de adecuación muestral de Kaiser-Meyer-Olkin. & \multicolumn{3}{c}{$\mathbf{0 , 5 6 1}$} \\
\hline Alfa de Cronbach & \multicolumn{3}{c}{$\mathbf{0 , 6 4 8}$} \\
\hline Prueba de esfericidad de Bartlett. & $X^{2}$ & gl & Sig. \\
\cline { 2 - 4 }
\end{tabular}

Método de extracción: Análisis de componentes principales. Método de rotación: Normalización Varimax con Kaiser. La rotación ha convergido en 6 iteraciones. 
TABLA 3- Análisis de componentes principales de las variables competitivas internas.

\begin{tabular}{|c|c|c|}
\hline \multirow[b]{2}{*}{ Variables } & \multicolumn{2}{|c|}{ Componente } \\
\hline & $\begin{array}{c}\text { Optimización } \\
\text { productiva }\end{array}$ & $\begin{array}{c}\text { Capacidad } \\
\text { industrial }\end{array}$ \\
\hline 1. Sistemas de conducción y poda & 0,876 & 0,031 \\
\hline 2. Políticas internas de producción limpia & 0,875 & $-0,061$ \\
\hline 3. Sistemas de control de calidad & 0,793 & 0,281 \\
\hline 4. Sistemas de optimización de servicios y suministros & 0,770 & 0,139 \\
\hline 5. Sistemas de control de inocuidad alimentaria & 0,729 & 0,101 \\
\hline 6. Integración vertical hacia atrás & 0,587 & 0,119 \\
\hline 7. Sistemas de mejoramiento de la productividad & $\mathbf{0 , 5 8 0}$ & 0,215 \\
\hline 8. Almacenaje & 0,055 & 0,883 \\
\hline 9. Tamaño e Infraestructuras de la empresa & $-0,218$ & $\mathbf{0 , 8 5 8}$ \\
\hline 10. Líneas de embalaje & 0,104 & $\mathbf{0 , 8 1 1}$ \\
\hline 11. Capacitación laboral & 0,224 & 0,667 \\
\hline 12. Automatización de los procesos productivos y comerciales & 0,271 & 0,650 \\
\hline 13. Sofisticación de redes de negocios & 0,276 & 0,558 \\
\hline Porcentaje de la varianza explicada por el factor & $32,51 \%$ & $27,12 \%$ \\
\hline Medida de adecuación muestral de Kaiser-Meyer-Olkin. & \multicolumn{2}{|c|}{0,589} \\
\hline Alfa de Cronbach & \multicolumn{2}{|c|}{$\mathbf{0 , 8 3 9}$} \\
\hline \multirow{2}{*}{ Prueba de esfericidad de Bartlett. } & $X^{2}$ & Sig. \\
\hline & 4449,299 & $\mathbf{0 , 0 0 0}$ \\
\hline
\end{tabular}

TABLA 4- Análisis de componentes principales de las estrategias competitivas.

\begin{tabular}{|c|c|c|}
\hline \multirow[b]{2}{*}{ Variables } & \multicolumn{2}{|c|}{ Componente } \\
\hline & $\begin{array}{c}\text { Optimización } \\
\text { de costos }\end{array}$ & $\begin{array}{c}\text { Gestión } \\
\text { comercial }\end{array}$ \\
\hline 1. Mano de obra & $\mathbf{0 , 8 5 1}$ & 0,111 \\
\hline 2. Especialización & $\mathbf{0 , 8 3 7}$ & 0,187 \\
\hline 3. Control de finanzas & 0,755 & $\mathbf{0 , 0 2 9}$ \\
\hline 4. Reducción de costos & 0,729 & 0,118 \\
\hline 5. Integración vertical & 0,656 & 0,495 \\
\hline 6. Control del flujo de productos & 0,638 & $\mathbf{0 , 5 3 0}$ \\
\hline 7. Canales de distribución del producto & $-0,089$ & 0,907 \\
\hline 8. Servicios & 0,101 & 0,821 \\
\hline 9. Estrategias de costos & 0,257 & 0,739 \\
\hline 10. Estrategias de compras & 0,261 & 0,648 \\
\hline 11. Relaciones comerciales con empresas matrices & 0,472 & 0,604 \\
\hline 12. Estrategias de ventas & 0,113 & 0,568 \\
\hline Porcentaje de la varianza explicada por el factor & $31,26 \%$ & $31,16 \%$ \\
\hline Medida de adecuación muestral de Kaiser-Meyer-Olkin. & \multicolumn{2}{|c|}{0,562} \\
\hline Alfa de Cronbach & \multicolumn{2}{|c|}{0,879} \\
\hline \multirow{2}{*}{ Prueba de esfericidad de Bartlett. } & $X^{2}$ & Sig. \\
\hline & 3428,393 & 0,000 \\
\hline
\end{tabular}




\section{CONCLUSIONES}

1- Al realizar la evaluación de los resultados de la investigación se puede concluir que la formulación de estrategias competitivas del sector exportador de la Región del Maule tiene dos constructos principales "optimización de costos" y la "gestión comercial", lo que indica estrategias de bajo costo, ya que los constructos encontrados se relacionan con la estrategia genérica "liderazgo en costos", que tiene por objetivo lograr economías de escala y optimizar todos los procesos productivos y comerciales del sector exportador de manzanas a los mercados asiáticos de China, Hong Kong, Taiwán e India.

2- Las variables de ventaja competitiva que tienen mayor relevancia para el sector exportador de manzanas a nivel interno son la "optimización productiva" y "capacidad industrial". En cuanto al nivel externo local, son "plataforma competitiva" y "externalidad productiva". En el caso del nivel externo global, las variables competitivas son "comercio exterior" y "mercados asiáticos". Tanto a nivel interno como externo las ventajas competitivas generadas indican un desarrollo de la industrialización y comercialización que se posiciona en los mercados de forma altamente competitiva.

3- Se considera relevante que en estudios futuros se analicen más detalladamente los constructos competitivos estratégicos encontrados por esta investigación, para así pasar de un estudio descriptivo-exploratorio a otras líneas investigativas más avanzadas.

\section{AGRADECIMIENTOS}

Los autores agradecen a la Dirección de Investigación y Perfeccionamiento de la Universidad Católica del Maule (UCM), por el apoyo financiero para esta investigación.

\section{REFERENCIAS}

BELROSE. Competitiveness update. World Apple Report, Pullman, v.17, n.10, p. 1- 16, 2010.

BISQUERRA, R. Introducción conceptual al análisis multivariante: un enfoque informático con los paquetes SPSS-X, BMDP, LISREL y SPAD. Barcelona: Promociones y Publicaciones Universitarias, 1989. v.1-2, p. 802.
CHACKO, T.; WACKER, J.; ASAR, M. Technological and human resource management practices in addressing perceived competitiveness in agribusiness firms. Agribusiness, United States of America, v. 13, n. 1, p. 93-105, 1997.

CHERNICK, M. R. Bootstrap methods: a practitioner's guide. Nueva York: Wiley \& Sons, 1999. p. 264.

CONTRERAS, P. Inteligencia competitiva para la industria de la fruta fresco chilena: análisis de escenarios. 2001. 68 f. Tesis (Licenciado en Ciencias Agrarias) - Facultad de Ciencias Agrarias. Universidad de Talca, Talca, 2001.

DECOFRUT. Export data 2008-2009 y 2009. 2010. Chile-Sudeste Asiático. Disponivel em: < http://www.fruitonline.com/expordata/default. aspx>. Acesso em: 14 mayo 2010.

DURAN, I.; SILVA, M. Industria de fruta fresca: estrategias empresariales. 1996. $164 \mathrm{f}$. Tesis (Licenciado en Ciencias de Administración de Empresas) - Facultad de Ciencias Empresariales, Universidad de Talca, Talca, 1996.

EFRON, B. Bootstrap methods: another look at the jackknife. The Annals of Statistics, Zürich, v.7, p. 1-26, 1979.

FJELDSTAD, O. D.; HAANAES, K. Strategy tradeoffs in the knowledge and network economy. Business Strategy Review, London, v.12, n.1, p. $1-10,2001$.

GARCÍA, J.E.; SERRANO, V. C. Competitividad y eficiencia: estudios de economía aplicada. Asociación de Economía Aplicada, Madrid, v.21, n.3, p. 423-450, 2003.

HERNANDEZ, R.;FERNANDEZ, C.;BAPTISTA, $P$ Metodología de la investigación. $4^{\text {th }}$ ed. México: Editorial McGraw-Hill, 2006. p.896.

KARANTININIS, K.; SAUER, J.; HARTLEY, W. Innovation and integration in the agri-food industry. Food Policy, Guilford, v.35, p.112-120, 2010.

KAISER, H.F. The varimax criterion for analytic rotation in factor analysis. Psychometrika, Williamshburg, v.23, p. 187-200, 1958. 
KINNEAR, T.; TAYLOR, J. Investigación de mercados. $5^{\text {th }}$ ed. México: McGraw-Hill Interamericana Editores, 2009. p. 874.

LEITNER, K. H.; GULDENBERG, S. Generic strategies and firm performance in SMEs: a longitudinal study of Austrian SMEs. Small Business Economics, Dordrecht, v.35, p. 169-189, 2010.

LOBOS, G. Estudio e identificación de clusters exportadores regionales (CER's) El caso de la Región del Maule. Chile: Bid-Direcon-Pro, 2007. p. 175 (Technical Reports)

LOBOS, G.; PETRI, J.L. Volatilidad de precios internacionales recibidos por los productores de kiwis y manzanas frescas chilenas. Revista Brasileira de Fruticultura, Jaboticabal, v.30, n.1, p. 133-139, 2008.

LUQUE, T. Análisis factorial: técnicas de análisis de datos en investigación de mercados. Madrid: Ediciones Pirámide, 2000. p. 39- 87.

MAMAQUI, X.; MEZA, L.; Albisu, L. Factores que influyen en la competitividad y estrategias de las empresas agroindustriales en Aragón, España. Agroalimentaria, Caracas, v.14, n.7, p. 69-88, 2002.

ORELlANA, C. Análisis competitivo de la fruta fresca chilena. 2009. 106f. Tesis (Licenciado en Ciencias Agrarias) - Universidad de Talca, Talca, 2009.

PORTER, M. Competitive strategy. techniques for analyzing industries and competitors. New York: The Free Press, 1982. p. 401.

PORTER, M. Estrategia y ventaja competitiva. Madrid: Editorial Deusto, 2006. p.222.

REARDON, T. Supermercados: Oportunidades y desafíos para la fruta chilena en economías emergentes. In: SEMINARIO REGIONAL DE PRODUCE MARKETING ASSOCIATION (PMA),2007,Santiago de Chile. Anais... Santiago de Chile: Filial Chilena de PMA, 2007. p.1-5.
ROJAS, G. Algunos aspectos de competitividad de la agricultura chilena. Chile Potencia Alimentaria. Santiago: Departamento de Economía Agraria, Pontificia Universidad Católica de Chile, 2007. p. 12-17.

SANTANA, A. Análise da competitividade sistêmica da indústria de madeira no Estado do Pará. Revista de Economia e Agronegócio, Viçosa, MG, v.1, n.2, p.205-230, 2004.

SCHWAB, K.; PORTER, M. The global competitiveness report 2008-2009. Switzerland: World Economic Forum Geneva, 2008. p.3-7.

SCHWAB, K. The global competitiveness report 2010-2011. Switzerland: World Economic Forum Geneva, 2010. p. 516.

STEWARD, P. Chile is the most competitive: the U.S. apple industry ranks fifth in global competitiveness, an agricultural economist calculates. Good Fruit Grower, Washington, v.59, n. 1, p. 12, 2008.

TAPIA, L. Estimación de la competitividad de los países exportadores de fruta del hemisferio sur a partir del índice de competitividad para el crecimiento en el período 2001-2004. IDESIA, Chile, v.24, n.2, p. 49-59, 2006.

TAPIA, L.; COOPER, T. Evaluación de la competitividad del kiwi neozelandés y chileno en el mercado de EE.UU. 1994-2004. IDESIA, Chile, v.24, n.3, p. 27-31, 2006.

THOMSON, A.; STRICKLAND, A.J. Dirección y administración estratégica. Buenos Aires: Editorial Addison-Wesley Iberoamericana, 1994. p. 309.

VILLACURA, J. Competitividad del sector exportador de fruta fresca: el caso de las manzanas. 2001. 81f. Tesis (Licenciado en Ciencias Agrarias) - Universidad de Talca, Talca, 2001.

WEHRENS, R.; PUTTER, H.; BUYDENS, L.M. The Bootstrap: a tutorial. Journal of Chemometrics and Intelligent Laboratory Systems, Amsterdam, v. 54, p. 35-52, 2000. 